\title{
SCLERITIS AS A SEQUEL OF HERPES OPHTHALMICUS
}

\author{
BY \\ G. G. PENMAN \\ LONDON
}

DURING the last year there seems to have been a great increase in the prevalence of herpes ophthalmicus, and of the cases I have seen, four have later developed scleritis, three of these being of a characteristic type, the fourth a sclero-keratitis.

References to this condition in the literature are few, and some of these rather vague.

The course of a typical case is as follows :-

About 2-3 months after an attack of herpes ophthalmicus, usually accompanied by some irido-cyclitis, the patient complains of redness and pain in the affected eye. On examination one or more nodules of a dark red colour, and about the size of a lentil, are found beneath the conjunctiva, which is much injected, and has a glossy surface. The nodules are very slow in subsiding, usually taking months to do so: gradually getting smaller and darker, almost plum-coloured, and finally disappearing, leaving sharply defined slaty grey areas in the sclera where they have been situated. The conjunctiva over these areas is freely movable, and apparently normal.

The iris is atrophic, often in patches, which are found in that part of the iris nearest to the slaty areas in the sclera. The pupil often remains quite inactive and dilated. The condition is liable to relapse, and fresh nodules may arise in areas of the sclera hitherto unaffected. The great majority of the cases are over 50 years of age. In addition to the characteristic condition described, in which there is no concurrent corneal involvement, a sclerokeratitis sometimes occurs.

Genet $^{1}$ quotes two cases, and divides the course of the condition into four stages:-(1) Patches of intense conjunctival hyperaemia with a glossy surface. (2) The appearance of raised nodules under the conjunctiva. (3) The return of the conjunctiva to its normal colour, leaving the nodules resembling those in episcleritis. (4) The disappearance of the nodules leaving flat slaty grey patches under the conjunctiva, which appears normal. He describes the lesions as essentially scleral, not corneal or conjunctival, and mentions that paralysis of the pupil is associated.

He thinks that the condition may be an affection of the sympathetic nerve twigs accompanying the first division of the fifth nerve. 
His first case is that of a man aged 60 years, who had herpes in January, 1926, and began to develop the nodules in March, 1926. The second is that of a man aged 60 years who had herpes in March, 1926, and developed nodules in April or afterwards, and was not seen again until November, when he had the typical slaty patches.

Lauber $^{2}$ quotes the case of a woman aged 57 years, who developed a swelling in the sclera six weeks after herpes. Her pupil was not paralyzed.

Thompson ${ }^{3}$ describes "herpetic eruptions " on the sclera, which appear as small reddish nodules, or larger nodules with a transparent centre. His four cases are as follows :-

(1) A man aged 41 years, who developed a nodule three weeks after the onset of herpes: the nodule left a discoloured patch behind it. The Wassermann reaction was positive in this case.

(2) A woman aged 36 years, who developed nodules two months after herpes.

(3) A man aged 66 years, who had three reddish swellings in the sclera 14 days after the onset of herpes.

(4) A woman aged 57 years, with nodules five months after herpes. Ten months after only slight discolouration of the sclera remained.

Wilbrand and Saenger ${ }^{4}$ mention scleritis as a complication of herpes ophthalmicus, but usually a sclero-keratitis. They quote Kendall, J..$^{5}$ as having found scleritis in three cases out of 17 of herpes ophthalmicus.

Meller ${ }^{6}$ mentions the condition, and quotes several of the above cases.

Verderame $^{7}$ describes a case of sclero-keratitis with serous cyclitis and raised tension coming on ten weeks after the onset of herpes ophthalmicus. He thinks that the condition is rheumatic in origin.

My own cases are as follows :-

Case I.-E. P., male, aged 63 years, attended St. Thomas's Hospital March 20, 1931, with a history of herpes ophthalmicus (left side) beginning 21 days before. Some scarring of the forehead was present, but no vesicles remained.

The right eye was normal. The left cornea was hazy, especially below, but did not stain. Both ciliary and conjunctival vessels were injected, and there was some iritis, with a few spots of fine K.P. The tension was normal.

He was admitted and treated with atropine and heat: the cornea cleared, the pupil remaining half dilated.

He was discharged March 28, 1931, R.V. $=6 / 6 \bar{c}+1 \cdot 0$ D. sph. L.V. $=6 / 12$ most $\overline{\mathrm{c}}+1 \cdot 0 \mathrm{sph}$. 
On April 7, 1931, two large patches of scleritis appeared at 5 and 7 o'clock, deep red in colour and covered by injected, glossy conjunctiva. Some atrophy was noted in the lower part of the iris with a network of delicate vessels coursing over the surface. Vision as before.

Case II.-S. G., female, aged 77 years, attended St. Thomas's Hospital June 23, 1930, with a history of herpes ophthalmicus (right side) three months previously, and inflammation of the right eye for a fortnight. There were no signs of active herpes.

Right Eye.-Vision $=6 / 18$. Much conjunctival injection. There were two large plum-coloured masses in the sclera at 7 and 11 o'clock, about the size of split peas. They did not reach to the limbus. The pupil was dilated and inactive. The iris was atrophic to the temporal side, especially in one patch at the point nearest to the lowest patch of scleritis. There were two tiny superficial scars in the cornea. Fine pigmented K.P. spots were present, and there were some lens striae. The fundus was normal.

Left Eye.-Vision $=6 / 36$. Considerable lens opacity. Otherwise normal. The patient was offered admission, but refused, so she was treated as an out-patient with atropine and diathermy, and given a salicylate mixture.

The condition continued much the same, except that two more nodules, rather smaller, appeared in the nasal half of the sclera.

The Sachs-Georgi reaction was negative, and no dental sepsis was present. Examination of the ears, nose, and throat was negative, except for some age deafness.

On July 27,1930 , she consented to come into hospital, and was an in-patient until August 23, 1930, when she was discharged, the nodules being much flatter.

Present State.-R.V. $=6 / 18$ pt. $\overline{\mathrm{c}}+1.5$ D. sph. Pupil dilated and inactive. Iris atrophy as before. Cornea as before, except that the K.P. has shrunk a little. Four slaty grey patches mark the nodules of scleritis. These are covered by conjunctiva which appears normal, and is freely movable over the patches. Lens and fundus as before.

Case III.-G. B., male, aged 66 years, attended the Royal Westminster Ophthalmic Hospital on April 5, 1930, with a history of herpes ophthalmicus (left side) commencing seven weeks previously. Well-marked scarring of the left side of the forehead and nose was present.

Right Eye.-V. $=6 / 9$ i glasses. Pupil active. Fundus normal.

Left Eye.-V. 6/60 $\bar{c}$ glasses. Pupil active. One tiny isolated staining point on the cornea. Fine K.P. Two patches of scleritis, one at 5 and one at 11 o'clock. Treated with atropine and hot bathings; the condition slowly improved, though two small fresh 
nodules appeared, one on June 14, 1930 and another on June 28, 1930.

The Wassermann reaction was negative, and no focal sepsis was found by examination of the teeth, and the ears, nose, and throat.

By the middle of July the nodules had settled in flat patches, and the patient was not seen again until January 31, 1931, when he attended with two large nodules at 3 and 5 o'clock. Two more nodules made their appearance in the next day or two, and he was admitted to hospital (February 2, 1931). He was treated with atropine and heat, given a salicylate mixture, and calcium chloride ionization to the left eye.

February 14, 1931. Discharged, Nodules almost flat, except that at 3 o'clock. Much K.P. still present. Fundus normal. Vision as before.

Case IV.-G. C., male, aged 63 years, admitted to the Royal Westminster Ophthalmic Hospital from an infirmary, April 5, 1930 , with a history of $\mathrm{R}$. herpes ophthalmicus since February 2, 1930. Some herpetic lesions were still active, the nasal branch of the nerve being involved. There was a large ulcerated patch the size of a shilling on the brow.

Right Eye.-V. = H.M. Patch of ulceration covering the whole of the lower half of the cornea. Large hypopyon. Complete external ophthalmoplegia and ptosis, also some weakness of the orbicularis.

Left Eye.-V. = $=6 / 6 \overline{\mathrm{c}}$ glasses. Normal in all respects.

The patient was treated with atropine and heat, and a partial lateral tarsorrhaphy performed.

On May 28, 1930, he was discharged, with the herpetic lesions quite healed, and the ocular and lid movements normal. There was a dense corneal scar obscuring the pupil. On June 25, 1930, a patch of scleritis appeared, continuous with the corneal scar. This cleared slowly, but did not leave a slaty grey area. The vessels in this area are still very numerous and dilated. R.V. = H.M. at 1 metre. L.V. $=6 / 6$ c glasses.

I am indebted to Mr. Hudson for permission to publish Case I, and to Mr. Doyne for Case II.

\section{REFERENCES}

1. Genet, L.-Lyon méd., Vol. XL, pp. 113-115, 1927.

2. Lauber.-Klin. Monatsbl. f. Augenheilk., Vol. LII, pt. I, pp. 284, 1914.

3. Thompson, G. W.-Brit. Jl. of Ophthal., Vol. II, p. 264, 1918.

4. Wilbrand and Saenger.-Die Neurologie des Auges., Bd. II, p. 170.

5. Kendall, J.-Inaug. Dissert., Zürich, 1880.

6. Meller, J.-Zeitsch. f. A ugenheilk., Vol. XLIII, pp. 457, 465, 1920.

7. Verderame, F.-Ann. di Ottal., Vol. XLII, pp. 772-789, 1913. 